\title{
ARTICLE
}

\section{Molecular basis for protection of ribosomal protein L4 from cellular degradation}

\author{
Ferdinand M. Huber ${ }^{1} \&$ André Hoelz ${ }^{1}$
}

Eukaryotic ribosome biogenesis requires the nuclear import of $\sim 80$ nascent ribosomal proteins and the elimination of excess amounts by the cellular degradation machinery. Assembly chaperones recognize nascent unassembled ribosomal proteins and transport them together with karyopherins to their nuclear destination. We report the crystal structure of ribosomal protein L4 (RpL4) bound to its dedicated assembly chaperone of L4 (Acl4), revealing extensive interactions sequestering 70 exposed residues of the extended RpL4 loop. The observed molecular recognition fundamentally differs from canonical promiscuous chaperone-substrate interactions. We demonstrate that the eukaryote-specific RpL4 extension harbours overlapping binding sites for $\mathrm{Acl} 4$ and the nuclear transport factor Kap104, facilitating its continuous protection from the cellular degradation machinery. Thus, Acl4 serves a dual function to facilitate nuclear import and simultaneously protect unassembled RpL4 from the cellular degradation machinery.

\footnotetext{
${ }^{1}$ Division of Chemistry and Chemical Engineering, California Institute of Technology, 1200 East California Boulevard, Pasadena, California 91125, USA. Correspondence and requests for materials should be addressed to A.H. (email: hoelz@caltech.edu).
} 
T he spatial separation of cytoplasmic protein translation and nucleolar ribosome biogenesis requires the nuclear import of $\sim 80$ nascent ribosomal proteins (RPs) through the nuclear pore complex (NPC) and subsequent export of preribosomal subunits into the cytoplasm ${ }^{1,2}$. These NPC-dependent nucleocytoplasmic transport events generate a layer of regulation that facilitates the dynamic adjustment of total ribosome numbers along with RP quality control and rapid degradation ${ }^{3-7}$. Ribosome biogenesis is temporally and spatially coordinated by $\sim 200$ trans-acting ribosome assembly factors that mediate the hierarchical assembly of pre-ribosomal subunits ${ }^{8}$. In addition, dedicated assembly chaperones assist ribosome biogenesis by recognizing and facilitating transport of nascent RPs to the pre-ribosome $e^{9-16}$.

In the mature ribosome, ribosomal proteins form multiple intricate interactions with both neighbouring RPs and ribosomal RNA (rRNA) ${ }^{17}$. Contacts with rRNA are mediated predominantly by electrostatic contacts between the phosphate backbone and arginine- and lysine-enriched motifs of RP elements located at their termini or within protruding loops ${ }^{17}$. The elongated $\sim 70$-residue loop of ribosomal protein L4 (RpL4) is devoid of secondary structure elements and extends deep into the large ribosomal subunit core, forming a series of interactions with rRNA and lines the peptide exit tunnel ${ }^{17,18}$. The mechanism by which nascent ribosomal proteins escape unfavourable interactions with nucleic acids, other RPs, and the cellular degradation machinery remains poorly understood. We have previously shown that the dedicated assembly chaperone Acl4 recognizes nascent RpL4, facilitates its nuclear import, and releases RpL4 on engaging RpL18 at the pre-ribosome ${ }^{10}$. Moreover, Acl4 is required for the soluble expression of newly synthesized RpL4 and for the protection of RpL4 from the Tom1-dependent cellular degradation machinery ${ }^{16,19}$. Therefore, we hypothesized that Acl4 could generate a protective environment for RpL4 by sequestering elongated RpL4 elements until its incorporation into the pre-ribosome.

Here, we report the 2.4- $\AA$ resolution crystal structure of RpL4 in complex with its dedicated ribosome assembly chaperone Acl4. The structure reveals an extensive interaction encompassing 70 exposed residues of the internal RpL4 loop that are sequestered by the concave Acl4 surface on complex formation. The observed binding mode differs dramatically from canonical chaperonesubstrate interactions that generally recognize short exposed hydrophobic peptide stretches. Despite the considerable binding interface in the Acl $4 \bullet \mathrm{RpL} 4$ complex, we identified a single Acl4 residue that abolishes RpL4 binding and may serve as an intrinsic weak spot for complex disassembly. Moreover, we determined the 3.0- $\AA$ resolution crystal structure of the karyopherin transport factor Kap104 in complex with the eukaryote specific RpL4 extension. Our structural and biochemical analysis demonstrates that the RpL4 extension possesses overlapping binding sites for a second Acl4 copy and Kap104. Whereas, unprotected RpL4 is targeted by the E3-ubiquitin ligase Tom1 for proteasomedependent degradation, interactions with Acl4 and Kap104 sequester Tom1 ubiquitination sites in the RpL4 loop and extension. Thus, ribosome assembly chaperones can serve dual functions by facilitating nuclear import while simultaneously protecting unassembled ribosomal proteins from the cellular degradation machinery.

\section{Results}

Crystal structure of the Acl4•RpL4 complex. We set out to identify the molecular basis of Acl4-dependent RpL4 sequestration and protection. We generated a biochemical interaction map between RpL4, its assembly chaperone Acl4, and its transport factor Kap104 and gained insight into the Acl4-RpL4 binding mode at the atomic level. Crystals of the Chaetomium thermophilum Acl4•RpL4 complex, which included the Acl4 TPR domain (residues 28 to 361 ) and the globular core of RpL4 $\left(\mathrm{RpL} 4{ }^{\mathrm{CORE}}\right)$ and the entire elongated loop (RpL4 $\left.{ }^{\mathrm{LOOP}}\right)$, diffracted to 2.4- $\AA$ resolution (Fig. 1a). The structure was solved by single-wavelength anomalous dispersion (SAD) using Seleno-Lmethionine (SeMet) labelled proteins. The final model was refined to $R_{\text {work }}$ and $R_{\text {free }}$ values of $19.1 \%$ and $22.7 \%$, respectively, with excellent stereochemistry (Table 1).

Acl4 adopts an $\alpha$-helical tetratricopeptide repeat (TPR) fold composed of seven TPRs $(\alpha \mathrm{A}-\alpha \mathrm{N})$ and a C-terminal flanking helix $(\alpha \mathrm{O})$ with an overall right-handed superhelical twist that accommodates the entire 70 -residue $\mathrm{RpL} 4{ }^{\mathrm{LOOP}}$ with its concave surface (Fig. 1b, Supplementary Fig. 1 and Supplementary Movie 1). Whereas, $\mathrm{RpL} 4 \mathrm{LOOP}$ forms numerous contacts with the Acl4 surface, RpL4 ${ }^{\mathrm{CORE}}$ contributes few additional interactions to the Acl4•RpL4 complex. Comparing the Acl4•RpL4 structure to our previously determined Acl4 apo structure revealed a conformational change on RpL4 $4^{\mathrm{LOOP}}$ binding, which is unusual for TPR domains (Fig. 1c) ${ }^{10}$. The longer central Acl4 helices $\alpha \mathrm{F}$ and $\alpha \mathrm{G}$ form a hinge between the $\mathrm{N}$-terminal $(\alpha \mathrm{A}-\alpha \mathrm{F})$ and C-terminal halves $(\alpha \mathrm{G}-\alpha \mathrm{O})$ of Acl4, which rotate as rigid bodies by $\sim 10^{\circ}$ from an open to a closed conformation on binding RpL4 (Fig. 1c).

RpL4 ${ }^{\mathrm{LOOP}}$ undergoes dramatic rearrangement on Acl4 binding. In the mature ribosome, the RpL4 ${ }^{\mathrm{LOOP}}$ adopts a remarkably elongated conformation, reaching deep into the rRNA core of the large ribosomal subunit, while the $\sim 100$-residue RpL4 extension, $\mathrm{RpL} 4{ }^{\mathrm{EXT}}$, extends $\sim 120 \AA$ over the ribosomal surface (Fig. $\left.1 \mathrm{~d}\right)^{17}$. Whereas, the conformation of $\mathrm{RpL} 4 \mathrm{CORE}$ remains largely unchanged, comparison of Acl4- and ribosome-bound RpL4 revealed a striking conformational change of the elongated $\mathrm{RpL} 4^{\mathrm{LOOP}}$ (Fig. 1d). Within the ribosome, RpL4 ${ }^{\mathrm{LOOP}}$ is fully extended and reaches $\sim 50 \AA$ into the centre of the large subunit ${ }^{17}$. In contrast, binding to Acl4 results in a great compaction of RpL4 $4^{\mathrm{LOOP}}$ by more than $\sim 15 \AA$, sequestering a maximum number of residues into the protective environment of the concave Acl4 surface (Fig. 1d). Acl4-binding induces the formation of an $\alpha$-helix within RpL4 ${ }^{\mathrm{LOOP}}(\alpha 3$, residues 89 to 97 ), which is entirely devoid of secondary structure elements in the context of the intact ribosome. Thus, both Acl4 and RpL4 undergo dramatic conformational changes on complex formation.

The majority of $\mathrm{RpL} 4{ }^{\mathrm{LOOP}}$ is buried by the concave Acl4 surface and involves several interactions formed by predominantly invariant Acl4 residues (Supplementary Figs 2-4). The extensive nature of the interactions is best illustrated by the sheer number of residues directly involved in Acl4-RpL4 ${ }^{\mathrm{LOOP}}$ binding: 42 out of $70 \mathrm{RpL} 4{ }^{\mathrm{LOOP}}$ residues and 87 out of 333 Acl4 residues (Fig. 2a; Supplementary Fig. 2). The interface is formed primarily by electrostatic interactions between the acidic Acl 4 surface and the basic RpL4 ${ }^{\mathrm{LOOP}}$ (Supplementary Fig. 4b,d). However, additional hydrophobic and $\pi$-stacking interactions contribute to the stability of the Acl4•RpL4 complex as well (Fig. 2a,b).

Acl4•RpL4 harbours an intrinsic weak spot for disassembly. Although, the extensive Acl4•RpL4 interface formed by a considerable number of direct interactions is ideally suited for substrate protection, this simultaneously represents a challenge for the eventual dismantling of the complex during ribosome biogenesis. To identify the underlying molecular mechanism, we employed a comprehensive structure- and conservation-guided mutagenesis approach with the goal of identifying Acl4 residues capable of triggering the disassembly of the Acl4-RpL4 interaction (Fig. 2a-e). Individual mutations of most of the invariant 
Acl4 residues proved to be insufficient to disrupt or even weaken the Acl4-RpL4 interaction (Supplementary Fig. 5). We next focused on two highly conserved interaction sites in the concave Acl 4 surface: the electrostatic interactions of Acl4 residues Glu180 and Glu212, both of which form a salt-bridge with RpL4 Arg108, and a hydrophobic pocket formed by Acl4 residues Tyr292 and Leu293, which engage RpL4 Phe101 (Fig. 2b). However, neither the Acl4 E180R/E212R charge-swap nor the Acl4 Y292A/L293A double mutation had a major effect on the interaction with RpL4 (Fig. 2b-d; Supplementary Fig. 5). In contrast, we identified a single charge-swap Acl4 mutation, E266R that abolished the Acl4-RpL4 ${ }^{\mathrm{LOOP}}$ interaction almost completely (Supplementary Fig. 5). Glu266 is located on the top surface of Acl4•RpL4 and forms hydrogen bonds with the mainchain amides of RpL4 residues Met100 and Phe101, thereby anchoring the C-terminal end of RpL4 helix $\alpha 3$ and compressing the RpL4 ${ }^{\mathrm{LOOP}}$ to the Acl4 surface (Fig. 2b). Introducing the identified Acl 4 mutants of the crystallized Chaetomium thermophilum protein into its Saccharomyces cerevisiae homologue established that the overall architecture of the Acl4•RpL4 complex is evolutionarily conserved (Fig. 2e; Supplementary Fig. 5). In fact, despite limited sequence conservation, C. thermophilum Acl4 is capable of forming a chimeric complex with $S$. cerevisiae RpL4 (Fig. 2e). These results suggest that the Glu266-mediated interactions constitute an intrinsic weak spot that is critical for Acl4•RpL4 complex disassembly. A structural comparison with the apo Acl4 reveals that disrupting these interactions upon engaging the preribosomal surface leads to the simultaneous relaxation of the Acl4

a

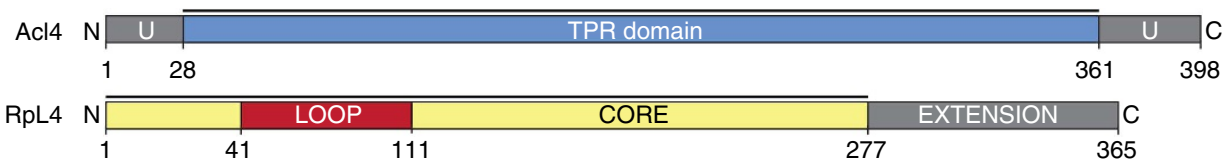

b
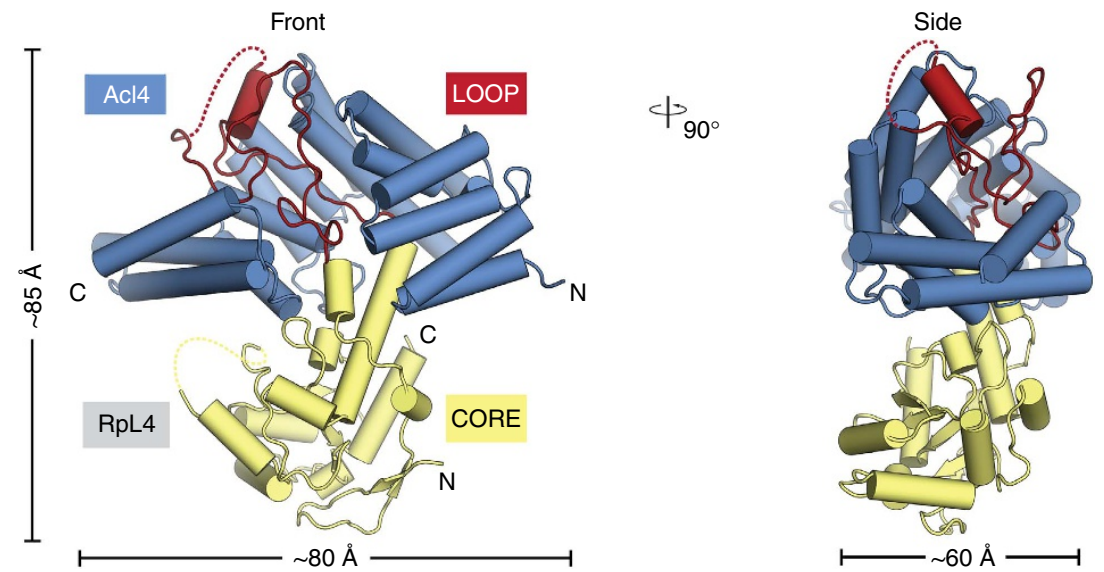

C
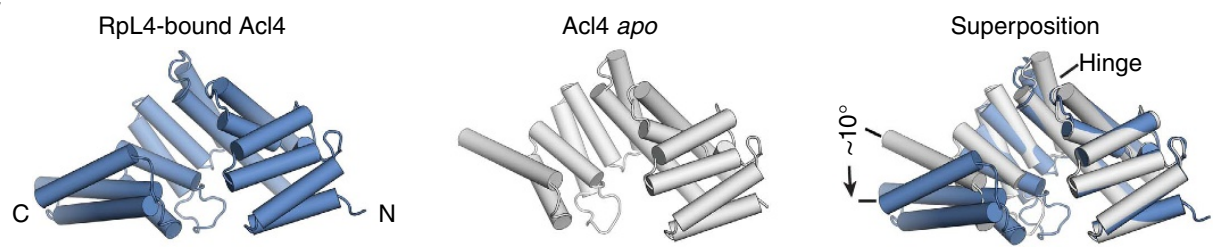

d

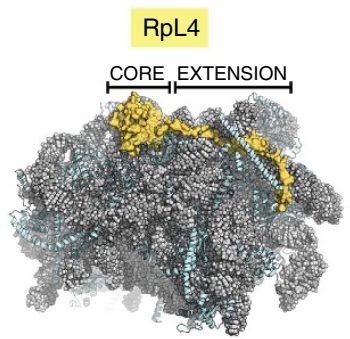

Acl4-bound RpL4

Ribosome-bound RpL4

Superposition
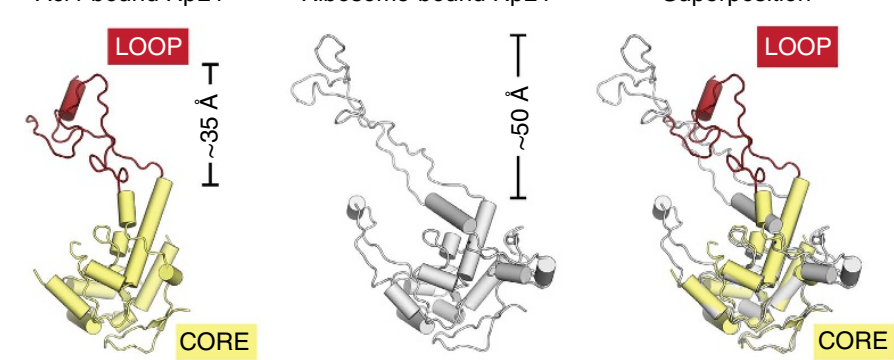

Figure 1 | Analysis of the Acl4•RpL4 structure. (a) Domain representation of Acl4 and RpL4 from Chaetomium thermophilum. Acl4: unstructured N- and C-terminal regions (dark grey); central TPR domain (blue). RpL4: core (yellow); loop (red); C-terminal extension (dark grey). Black bars represent crystallized fragments. (b) Crystal structure of the Chaetomium thermophilum Acl4•RpL4 complex, shown in cartoon representation. $\mathrm{A} 90^{\circ}$ rotation is shown on the right. Colouring is according to panel a. (c) Superposition of RpL4-bound Acl4 (blue) with Acl4 apo (grey) (PDB ID 4YNV) ${ }^{10}$. (d) Cartoon representation of the S. cerevisiae large ribosomal subunit (PDB ID 4V88) showing RNA (grey), proteins (teal), and RpL4 (yellow) ${ }^{17}$. Superposition of Acl4-bound RpL4 with ribosome-bound RpL4 (grey). 
TPR domain and elongation of $\mathrm{RpL} 4^{\mathrm{LOOP}}$, reminiscent of a spring-loaded mechanism (Supplementary Movie 2).

To validate the physiological relevance of our findings on the mechanism of Acl4-RpL4 binding and disassembly, we generated an S. cerevisiae Acl4 deletion (acl44) strain and analysed various Acl 4 mutants. Deletion of TPR1 and the acidic C-terminal region of Acl4 caused a growth defect at $37^{\circ} \mathrm{C}$, as did mutation of the conserved hydrophobic pocket with Tyr292 and Leu293 to alanines, consistent with the biochemical findings. In contrast, the E266R mutant displayed a severe growth defect, identical to the acl44 phenotype, demonstrating the physiological relevance of closely anchoring RpL4 ${ }^{\mathrm{LOOP}}$ to the Acl4 surface. Notably, the Acl4 E266R mutation exhibited identical behaviour in size exclusion chromatography, confirming that the observed effect was not caused by improper Acl4 E266R protein folding (Supplementary Fig. 6). Surprisingly, the E180R/E212R double charge-swap mutation of the electrostatic binding site, which only moderately affected Acl4-RpL4 binding, also caused a substantial growth defect at all analysed temperatures, suggesting a role of this binding site for the proper release of RpL4 into the maturing ribosome. Notably, all Acl4 variants were expressed at similar levels and predominantly localized to the nucleus with the RpL4-binding deficient mutants only displaying a slight increase in cytoplasmic localization (Fig. 2g; Supplementary Fig. 7).

Acl4 and Kap104 share an overlapping binding site on RpL4 ${ }^{\mathrm{EXT}}$. By employing a more sensitive size exclusion chromatography assay, we identified an additional interaction between Acl4 and $\mathrm{RpL}^{\mathrm{EXT}}{ }^{\text {, which }}$ was previously missed in GST pull-down and yeast two-hybrid assays ${ }^{10,16}$. We found that the heterodimeric Acl4•RpL4 complex is capable of interacting with an additional Acl 4 molecule resulting in the formation of a heterotrimeric Acl4•RpL4•Acl4 complex with a 2:1 stoichiometry that is evolutionarily conserved (Fig. 3a; Supplementary Fig. 8a). Mapping of the binding site established that RpL4 ${ }^{\mathrm{EXT}}$ is necessary and sufficient for binding of the second Acl4 copy (Fig. 3b). Further truncation analysis identified an 18-residue region encompassing residues $311-328$ of $\mathrm{RpL} 4{ }^{\mathrm{EXT}}$ that is required for Acl $4 \bullet$ RpL4•Acl 4 complex formation. Alanine substitution of the three highly conserved basic residues in this region, Lys316, Lys317 and Arg321, substantially reduced binding of the second Acl4 copy to Acl4•RpL4 (Fig. 3b-d). Notably, we observed no Acl4 exchange from the RpL4 ${ }^{\mathrm{LOOP}}$ binding site in the conditions of the performed pull-down experiments, demonstrating that the Acl4•RpL4 heterodimer is very stable in solution.

Previously, we found that the transport factor Kap104 binds to Acl4•RpL4 to form a heterotrimeric complex with equimolar stoichiometry ${ }^{10}$. Further mapping revealed that two distinct regions in Acl4•RpL4 are sufficient for Kap104 binding, one located in RpL4 ${ }^{\mathrm{EXT}}$ and another in the basic unstructured 28-residue N-terminal region of Acl4 (Fig. 3h; Supplementary Fig. 9). Consistently, the binding between Acl4•RpL4 and Kap104 is abolished when both regions are deleted (Supplementary Fig. 9c). RpL4 ${ }^{\mathrm{EXT}}$ harbours a canonical basic phenylalaninetyrosine nuclear localization signal (PY-NLS) and alanine mutagenesis confirmed that all canonical elements of its consensus sequence are critical for the Kap104-RpL4 ${ }^{\text {EXT }}$ interaction (Fig. 3e $)^{20}$. Because Acl4 and Kap104 bind to overlapping sites in $\mathrm{RpL} 4^{\mathrm{EXT}}$ and $\mathrm{Acl} 4 \bullet \mathrm{RpL} 4$ possesses a second Kap104 binding site, we tested whether Kap104 is able to displace the $\mathrm{RpL} 4{ }^{\mathrm{EXT}}$-bound Acl4 copy. Indeed, in a competition experiment Kap104 replaced the RpL4 ${ }^{\mathrm{EXT}}$-bound Acl4 copy to form an Acl4•RpL4•Kap104 complex (Fig. 3f). As expected, RanGTP released Acl4•RpL4 from this nuclear import heterotrimer (Fig. $3 \mathrm{~g}$ ). In addition, the nuclear import

\section{Table 1 | Data collection and refinement statistics.}

\begin{tabular}{|c|c|c|}
\hline \multicolumn{3}{|l|}{ Data Collection } \\
\hline Protein & $\mathrm{Acl} 4^{28-361} \cdot \mathrm{RpL}^{1-277}$ & hsKap104•RpL4 308-332 \\
\hline PDB ID & $5 T Q B$ & 5TQC \\
\hline Synchrotron & $\mathrm{SSRL}^{\star}$ & $S S R L^{*}$ \\
\hline Beamline & $12-2$ & $12-2$ \\
\hline Space group & $P 2,2,2$ & $\mathrm{P} 2{ }_{1} 2,2$ \\
\hline \multicolumn{3}{|l|}{ Cell dimensions } \\
\hline$a, b, c(\AA)$ & $121.0,127.9,42.7$ & $68.6,130.7,174.2$ \\
\hline$\alpha, \beta, \gamma\left(^{\circ}\right)$ & $\begin{array}{c}\text { 90.0, 90.0, } 90.0 \\
\text { Se Peak }\end{array}$ & $\begin{array}{c}\text { 90.0, } 90.0,90.0 \\
\text { Native }\end{array}$ \\
\hline Wavelength $(\AA)$ & 0.9792 & 1.0000 \\
\hline Resolution $(\AA)$ & $50.0-2.4$ & $50.0-3.0$ \\
\hline$R_{\text {merge }}(\%)^{\dagger}$ & $8.9(99.0)$ & $9.3(192.7)$ \\
\hline$R_{\text {pim }}(\%)^{\dagger}$ & $2.6(28.5)$ & $2.7(53.5)$ \\
\hline$<l>|<\sigma l\rangle^{\dagger}$ & $13.6(1.9)$ & $20.7(1.6)$ \\
\hline $\mathrm{CC}_{1 / 2}^{\dagger}$ & $99.9(89.8)$ & $99.9(75.7)$ \\
\hline Completeness $(\%)^{\dagger}$ & $99.1(99.1)$ & $99.8(99.9)$ \\
\hline No. of observations & 338,722 & 425,167 \\
\hline $\begin{array}{l}\text { No. of unique } \\
\text { reflections }\end{array}$ & $49,797(8,039)$ & $32,119(5,078)$ \\
\hline Redundancy ${ }^{\dagger}$ & $6.8(6.6)$ & $13.2(13.7)$ \\
\hline \multicolumn{3}{|l|}{ Refinement } \\
\hline Resolution $(\AA)$ & $50.0-2.4$ & $50.0-3.0$ \\
\hline No. of reflections & 49,767 & 32,065 \\
\hline $\begin{array}{l}\text { No. of reflections } \\
\text { test set }\end{array}$ & $2,505(5.0 \%)$ & $1,606(5.0 \%)$ \\
\hline$R_{\text {work }} / R_{\text {free }}(\%)$ & $19.1 / 22.7$ & $20.8 / 23.8$ \\
\hline No. atoms & 4,605 & 6,750 \\
\hline Protein & 4,510 & 6,750 \\
\hline Ligands & 39 & 0 \\
\hline Water & 56 & 0 \\
\hline \multicolumn{3}{|l|}{$B$-factors } \\
\hline Protein & 73 & 103 \\
\hline Ligands & 87 & $\mathrm{~N} / \mathrm{A}$ \\
\hline Water & 60 & N/A \\
\hline \multicolumn{3}{|l|}{ r.m.s.d. } \\
\hline Bond lengths $(\AA)$ & 0.004 & 0.002 \\
\hline Bond angles $\left({ }^{\circ}\right)$ & 0.7 & 0.7 \\
\hline \multicolumn{3}{|l|}{ Ramachandran plot ${ }^{\ddagger}$} \\
\hline Favored (\%) & 96.7 & 97.4 \\
\hline Outliers (\%) & 0.0 & 0.0 \\
\hline \multicolumn{3}{|l|}{ MolProbity } \\
\hline Clash score & 1.98 & 1.77 \\
\hline MolProbity score & 1.17 & 1.05 \\
\hline
\end{tabular}

adaptor Kap- $\alpha$ was also able to form a heterotrimeric Acl4•RpL4•Kap- $\alpha$ nuclear import complex, indicating that multiple karyopherins are capable of transporting Acl4•RpL4 to the nucleus (Supplementary Figs 8d,9). However, Kap104 displaced Kap- $\alpha$ from the Acl $4 \bullet$ RpL4๑Kap- $\alpha$ heterotrimer in direct competition experiments, suggesting that Kap104 is the primary nuclear import factor for Acl4 $\bullet$ RpL4 (Supplementary Fig. 8e).

Acl4 and Kap104 protect nascent RpL4 from degradation. We previously described a novel pathway for excess ribosomal protein quality control (ERISQ) involving the E3 ubiquitin ligase Tom1, which marks excess ribosomal proteins for proteasomedependent degradation ${ }^{19}$. RpL4 ${ }^{\mathrm{LOOP}}$ residue Lys56 along with $\mathrm{RpL}^{\mathrm{EXT}}{ }^{\mathrm{ET}}$ residues Lys310 and Lys340 were identified as Tom1 
a

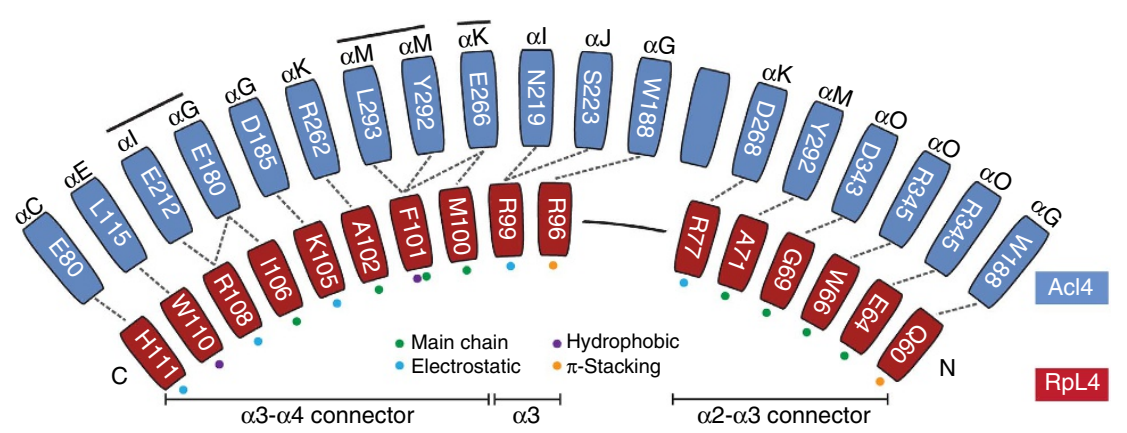

b

Electrostatic interactions

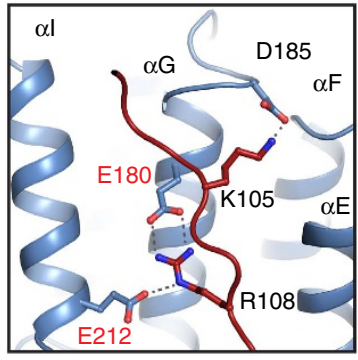

Hydrophobic pocket

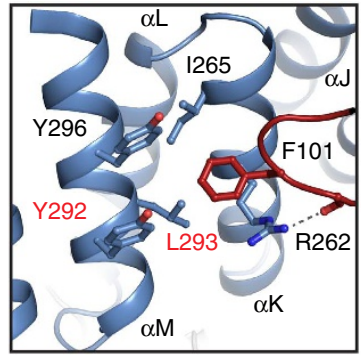

Critical E266 interaction

C

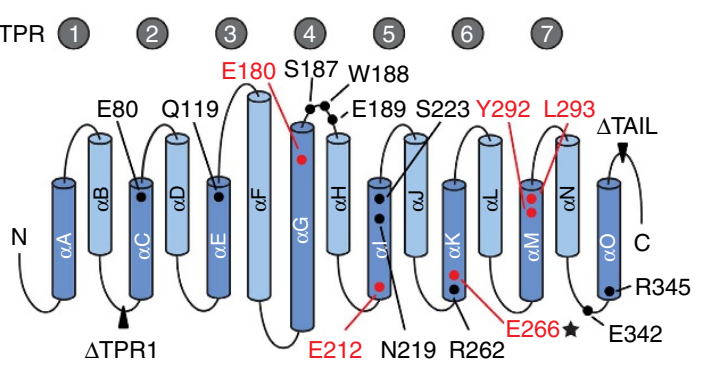

d

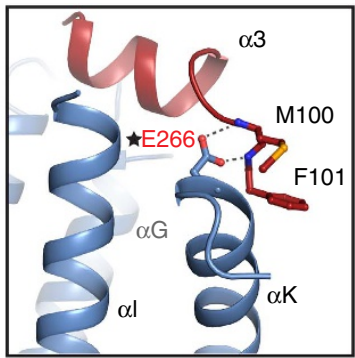

e

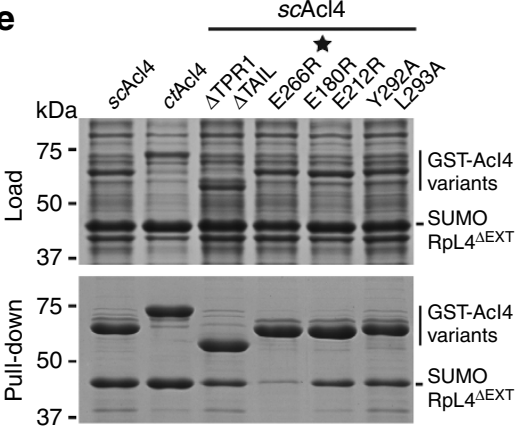

g

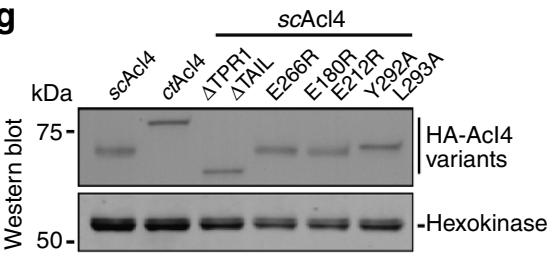

f

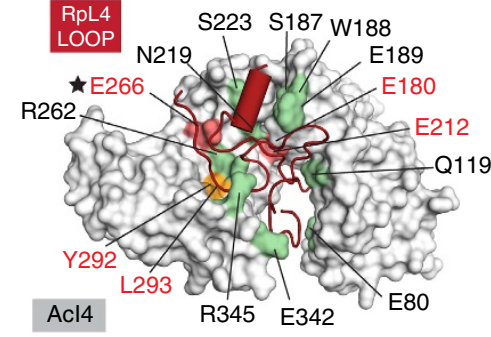

SDC-Leu

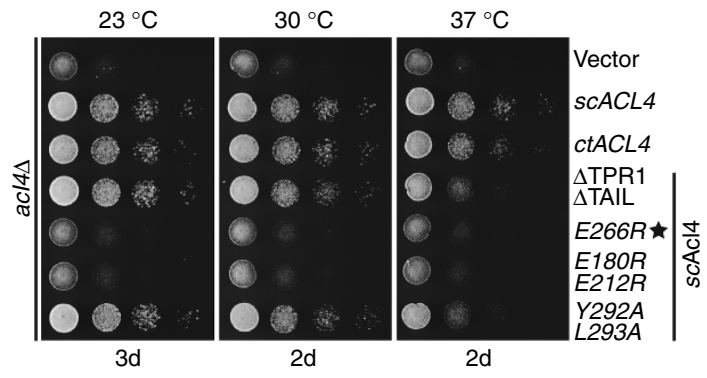

Figure 2 | Acl4-RpL4 interaction analysis. (a) Schematic representation of the Acl4-RpL4 interface, coloured as in Fig. 1. Coloured dots indicate the interaction type between depicted residues. (b) Acl4•RpL4 interaction hotspots. Boxes show three interactions between RpL4 (red) and Acl4 (blue) in cartoon representation. (c) Schematic representation of the Acl4 TPR domain fold architecture. The positioning of RpL4 interaction residues is indicated. (d) Mutational analysis of the Acl4-RpL4 interaction. Acl4 (grey) and RpL4 ${ }^{\mathrm{LOOP}}$ (red) are shown in surface and cartoon representation, respectively. Mutated Acl 4 residues are plotted on the surface and coloured according to effect on RpL4 binding: green, no effect; orange, medium effect; and red, strong effect. (e) Interaction analysis of Acl4 and RpL4 ${ }^{\Delta \mathrm{EXT}}$. Pull-down interaction analysis between S. cerevisiae GST-Acl4 variants (bait) and RpL4 ${ }^{\Delta \mathrm{EXT}}$. Loaded (top) and pulled-down (bottom) fractions are indicated and Acl4 mutations are depicted above each lane. (f) Growth analysis of Acl4 variants. Residue numbering is according to $\mathrm{C}$. thermophilum Acl4. (g) Western blot analysis of the expression levels of Acl4 variants in S. cerevisiae. HA-tagged Acl4 variants and the hexokinase loading control were detected with anti-HA and anti-hexokinase antibodies, respectively. 
a

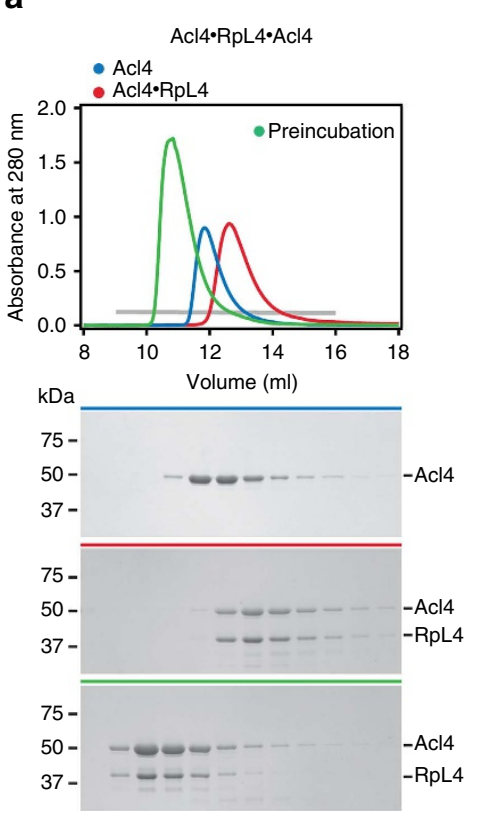

b

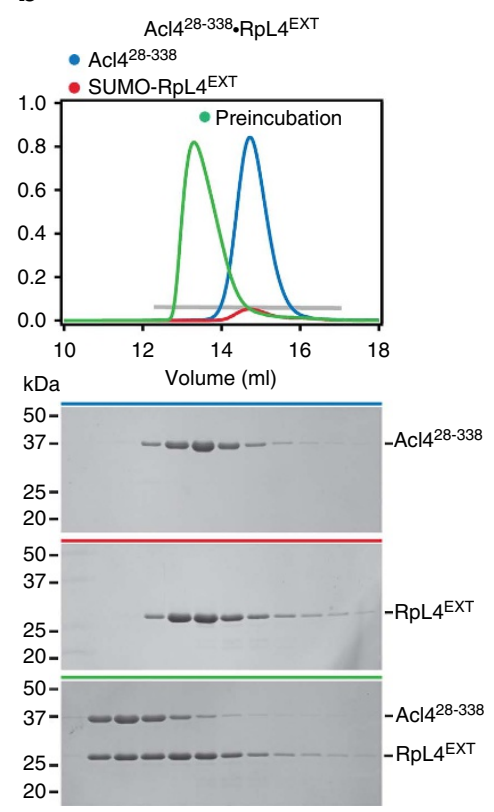

c

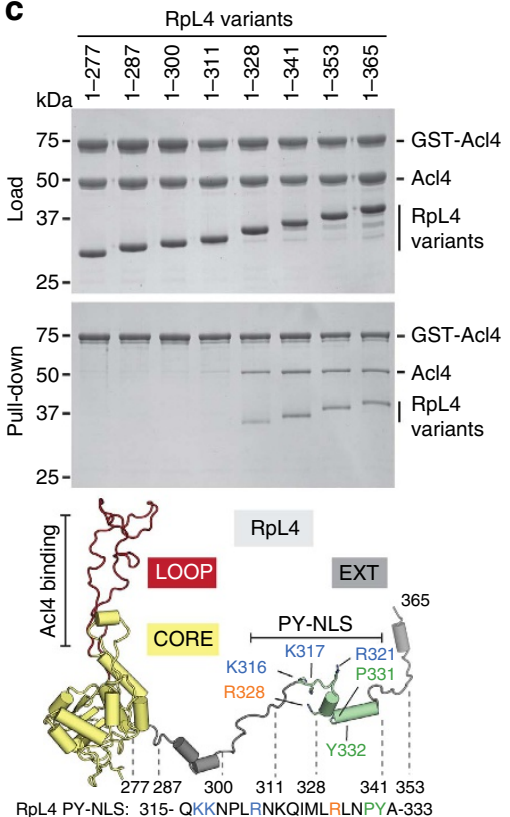

d

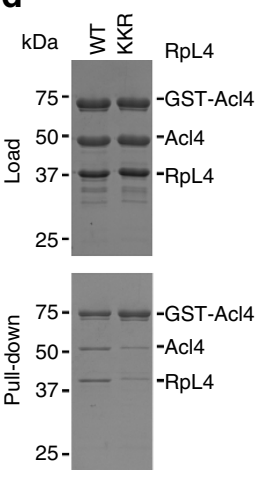

e

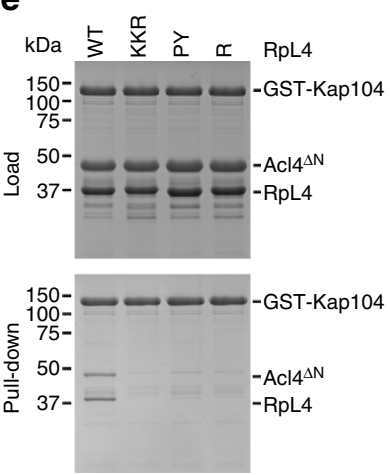

f

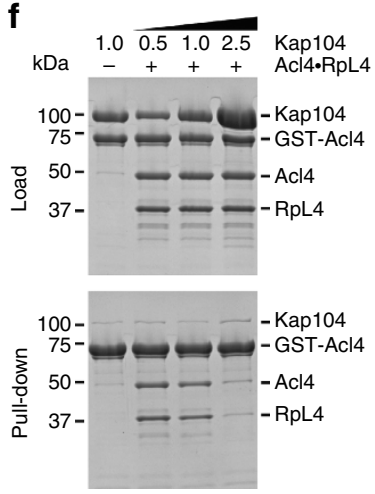

g

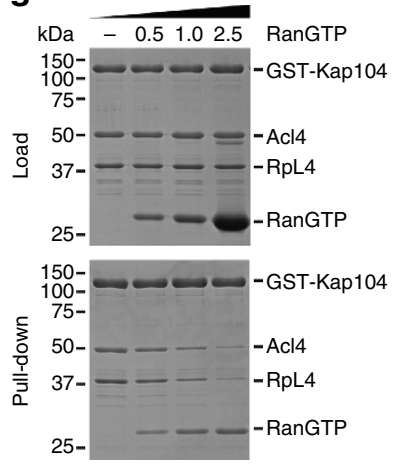

h

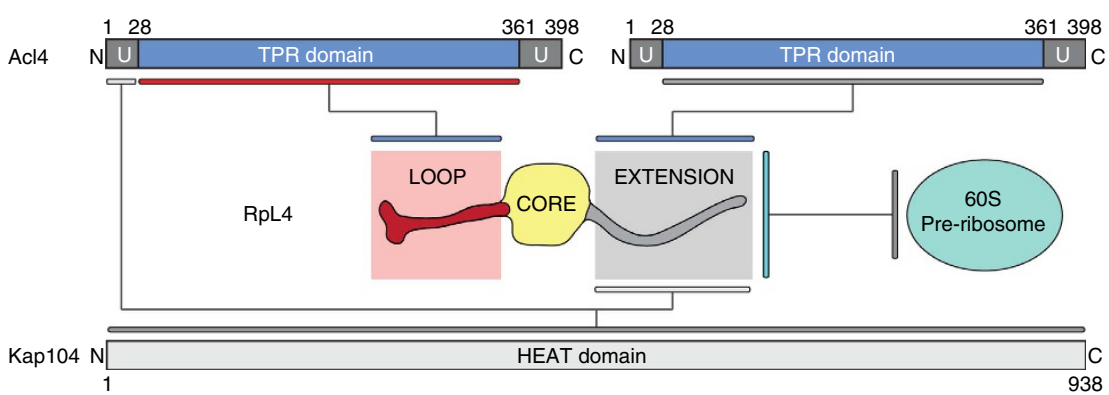

Figure 3 | Biochemical Acl4•RpL4•Kap104 interaction map. (a,b) Size exclusion chromatography (SEC) analysis of Acl4, Acl4•RpL4, and SUMO-RpL4 ${ }^{\mathrm{EXT}}$. SEC profiles of proteins or protein complexes are shown individually (blue and red) and after preincubation (green). (c) GST pull-down of pre-purified GST-Acl4 and Acl4•RpL4 C-terminal truncation variants. Loaded (top) and pulled-down (bottom) fractions are shown. Cartoon representation of RpL4 with RpL4 ${ }^{\mathrm{LOOP}}, \mathrm{RpL} 4{ }^{\mathrm{CORE}}$, and RpL4 ${ }^{\mathrm{EXT}}$ coloured as in Fig. 1a. The basic PY-NLS is coloured in green and the analysed fragment boundaries are indicated. As reference, the primary sequence of the basic PY-NLS and the consensus residues are shown. (d) GST pull-down with pre-purified GST-Acl4 and Acl4•RpL4 variants. (e) GST pull-down with pre-purified GST-Kap104 and Acl4 ${ }^{\Delta N} \bullet$ RpL4 variants. Labelling indicates RpL4 variants (WT, wild type; KKR, K316A/K317A/R321A; PY, P331A/Y332A; R, R328A). (f) GST pull-down with pre-assembled Acl4•RpL4•GST-Acl4 and increasing amounts of Kap104. (g) GST pull-down with pre-assembled Acl4•RpL4•GST-Kap104 and increasing amounts of RanGTP. (h) Schematic representation of the Acl4•RpL4•Kap104 interaction map.

recognition sites, which were ubiquitinated in the absence of Acl4 and Kap104 (Fig. 4a) ${ }^{19}$. The crystal structure of Acl4•RpL4 now shows that Lys56 is located in the highly conserved RpL4 ${ }^{\mathrm{LOOP}}$ and is sequestered by the Acl4 surface, thus shielded from Tom1mediated ubiquitination (Fig. 4b). These findings demonstrate that in the RpL4-binding deficient Acl4 E266R mutant Lys56 in
$\mathrm{RpL} 4{ }^{\mathrm{LOOP}}$ is not sequestered by Acl4 and therefore is a target for Tom1-dependent ubiquitination. Thus, the growth defect observed in the Acl4 E266R mutant likely is the consequence of Tom1-dependent RpL4 ubiquitination and degradation, resulting in reduced soluble levels of RpL4 and in turn of $60 \mathrm{~S}$ preribosomal particles ${ }^{10,16}$. 
a

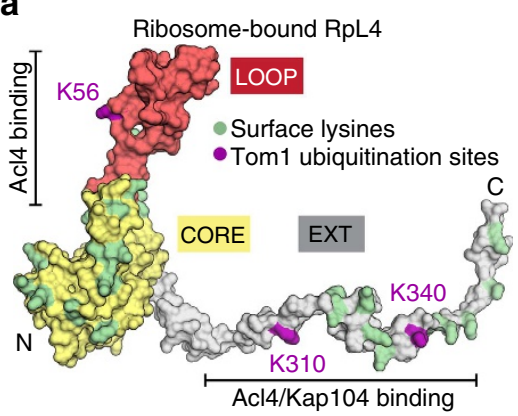

b

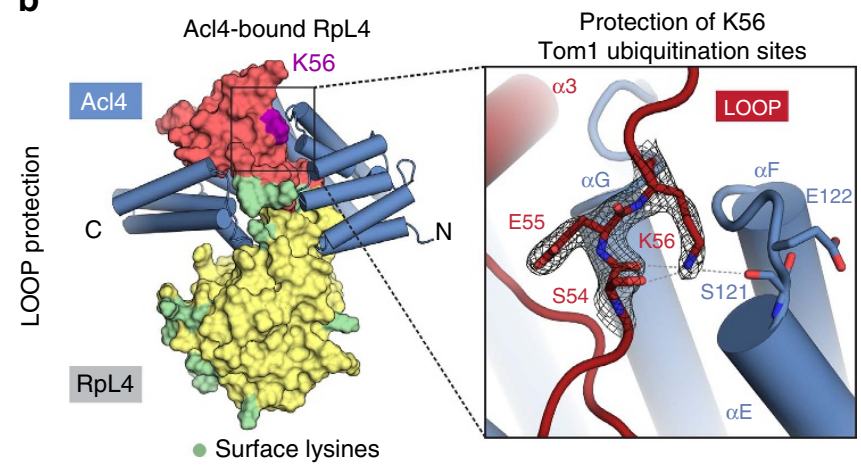

C

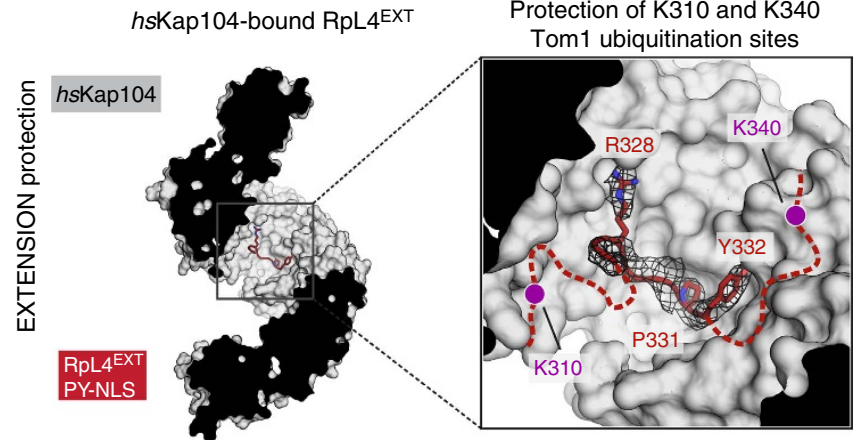

Figure 4 | Shielding of Tom1 ubiquitination sites. (a) Surface representation of ribosome-bound RpL4 (PDB ID 4V88) ${ }^{17}$. Acl4 and Kap104 binding sites are indicated with black bars. (b) Acl4-bound RpL4 (coloured as in Fig. 1a) and Acl4 (blue) are shown in surface and cartoon representation, respectively. The inset marks the Tom 1 ubiquitination site that is illustrated in detail on the right. Acl4 (blue) and RpL4 (red) and critical residues highlighted in stick representation with a section of the final $2\left|\mathrm{~F}_{\mathrm{o}}\right|-\left|\mathrm{F}_{\mathrm{c}}\right|$ electron density map contoured at $1.0 \sigma$. (c) Crystal structure of the hsKap104•RpL4 ${ }^{\mathrm{EXT}}$ complex. The inset marks the location of the Kap104 PY-NLS binding site that is illustrated in detail on the right. The residues of the PY-NLS consensus sequence, Arg328, Pro331 and Tyr332 are highlighted in stick representation with a section of the final $2\left|\mathrm{~F}_{\mathrm{o}}\right|-\left|\mathrm{F}_{\mathrm{c}}\right|$ electron density map contoured at $1.0 \sigma$. Magenta circles indicate the approximate location of RpL4 residues $\mathrm{K} 310$ and $\mathrm{K} 340$ that are ubiquitinated by Tom1 in the absence of Kap104.

To explore whether RpL4 ${ }^{\mathrm{EXT}}$ residues Lys310 and Lys340 are protected by Kap104 in a similar fashion, we determined the crystal structure of Kap104 in complex with $\mathrm{RpL} 4{ }^{\mathrm{EXT}}$ to $3.0 \AA$ resolution. The Kap104•RpL4 ${ }^{\mathrm{EXT}}$ structure revealed that the PY-NLS of RpL4 ${ }^{\mathrm{EXT}}$ engages the concave surface of Kap104 in the same binding mode as previously established for other PY-NLS sequences ${ }^{20}$. Upon Kap104 binding to RpL4 ${ }^{\mathrm{EXT}}$ and formation of a nuclear import complex both Tom1-modification sites of RpL4 ${ }^{\mathrm{EXT}}$ are sequestered by the concave Kap104 surface, consistent with our previous protection results of an in vitro Tom1 ubiquitination assay (Fig. $4 \mathrm{c})^{19}$.

In summary, these results together with our previous findings allow us to propose a model of the entire RpL4 life cycle (Fig. 5): Nascent RpL4 binds two Acl4 copies, one via RpL4 $4^{\mathrm{LOOP}}$ and another via RpL4 ${ }^{\mathrm{EXT}}$. Kap104 replaces one Acl4 copy and shuttles Acl4•RpL4 across the nuclear envelope. Once in the nucleus, Kap104 releases RpL4 ${ }^{\mathrm{EXT}}$ on RanGTP binding allowing the rebinding of a second Acl4 copy from the nuclear Acl4 pool. RpL4 release from Acl4 and ribosome incorporation is dependent on the interaction of $\mathrm{RpL} 4{ }^{\mathrm{EXT}}$ with RpL18 and is triggered by relaxation of the presumably spring-loaded Acl4^RpL4 complex at the pre-60S ribosomal subunit (Fig. $5 \mathrm{a})^{10}$. Whereas, unprotected RpL4 is recognized and ubiquitinated by the E3 ligase Tom1, followed by its proteasome-dependent degradation, protection of RpL4 by Acl4 and Kap104 generates a pool of RpL4 available for ribosome biogenesis ${ }^{16,19}$. Thus, ribosome assembly chaperones not only facilitate nuclear import and pre-ribosome incorporation of their ribosomal protein substrates, but are also essential for their protection from the cellular degradation machinery (Fig. 5b). It remains an open question how Acl4 and other ribosome assembly chaperones return to the cytoplasm after their substrate RPs are incorporated in the pre-ribosomal particle and whether this occurs in a karyopherin-dependent manner. However, the presence of only sub-stoichiometric amounts of Acl4 in the cell strongly suggests that Acl4 shuttles between nucleus and cytoplasm. Furthermore, because the NPC allows passive diffusion of small proteins with a mass of less than $\sim 40 \mathrm{kDa}$, the re-export of free Acl4 may not require a dedicated transport factor. The next important steps will be to identify and characterize the assembly chaperones for the remaining $\sim 70$ ribosomal proteins to establish whether the principles identified for Acl4 are conceptually similar. Additionally, the development of an in vitro ribosomal assembly system will be essential for the elucidation of the complex interplay of chaperoned ribosomal proteins, the cellular degradation machinery, and the maturing pre-ribosomal particle.

Unlike promiscuous folding chaperones that recognize exposed short hydrophobic secondary structure elements, Acl4 serves a dedicated sequestering function and harbours an intrinsic trigger for RpL4 release. Thus, the Acl4-RpL4 interaction constitutes a prototype for a dedicated assembly chaperone-substrate interaction that exerts multiple functions. We envision that a similar mechanism is employed by other ribosomal assembly chaperones and by assembly factors of other multi-component macromolecular machineries.

\section{Methods}

Bacterial and yeast expression constructs. S. cerevisiae and C. thermophilum DNA fragments of Acl4, RpL4, Kap104 and Kap- $\alpha$ and of Homo sapiens Ran were amplified by PCR and ligated into bacterial expression vectors pGEX-6P-1 (GE Healthcare), a modified pET28a and pETDuetl vector (both Novagene) that contained an $\mathrm{N}$-terminal $\mathrm{His}_{6}$-SUMO (small ubiquitin-like modifier) tag (pET28a-SUMO, pETDuet1-SUMO), and a modified pET28a vector (Novagene) containing an N-terminal $\mathrm{His}_{6}$ tag followed by a PreScission cleavage site ${ }^{21,22}$. The expression construct of H. sapiens Kap104 in which the internal loop residues 337-367 were replaced with a GGSGGSG linker was a gift from Yuh Min Chook ${ }^{20}$ Acl4 and RpL4 variants were amplified by PCR and ligated into yeast expression vectors pRS415, pRS415-mCherry, pRS415-HA-mCherry and pRS413-eGFP. Mutants were generated using QuikChange mutagenesis (Stratagene) and confirmed by DNA sequencing. Details of all bacterial and yeast expression constructs are summarized in Supplementary Tables 1 and 2.

Protein expression and purification. Bacterial expression constructs were transformed in Escherichia coli BL21-CodonPlus(DE3)-RIL cells (Stratagene) and grown in $\mathrm{LB}$ medium to an $\mathrm{OD}_{600}$ of $\sim 0.6$ before induction with $0.5 \mathrm{mM}$ isopropyl $\beta$-D-thiogalactoside (IPTG). Cultures containing C. thermophilum protein expression constructs were grown for $18 \mathrm{~h}$ at $23^{\circ} \mathrm{C}$, while S. cerevisiae and H. sapiens proteins were expressed for $18 \mathrm{~h}$ at $18^{\circ} \mathrm{C}$. Cells were harvested by centrifugation 
a

Ribosome biogenesis

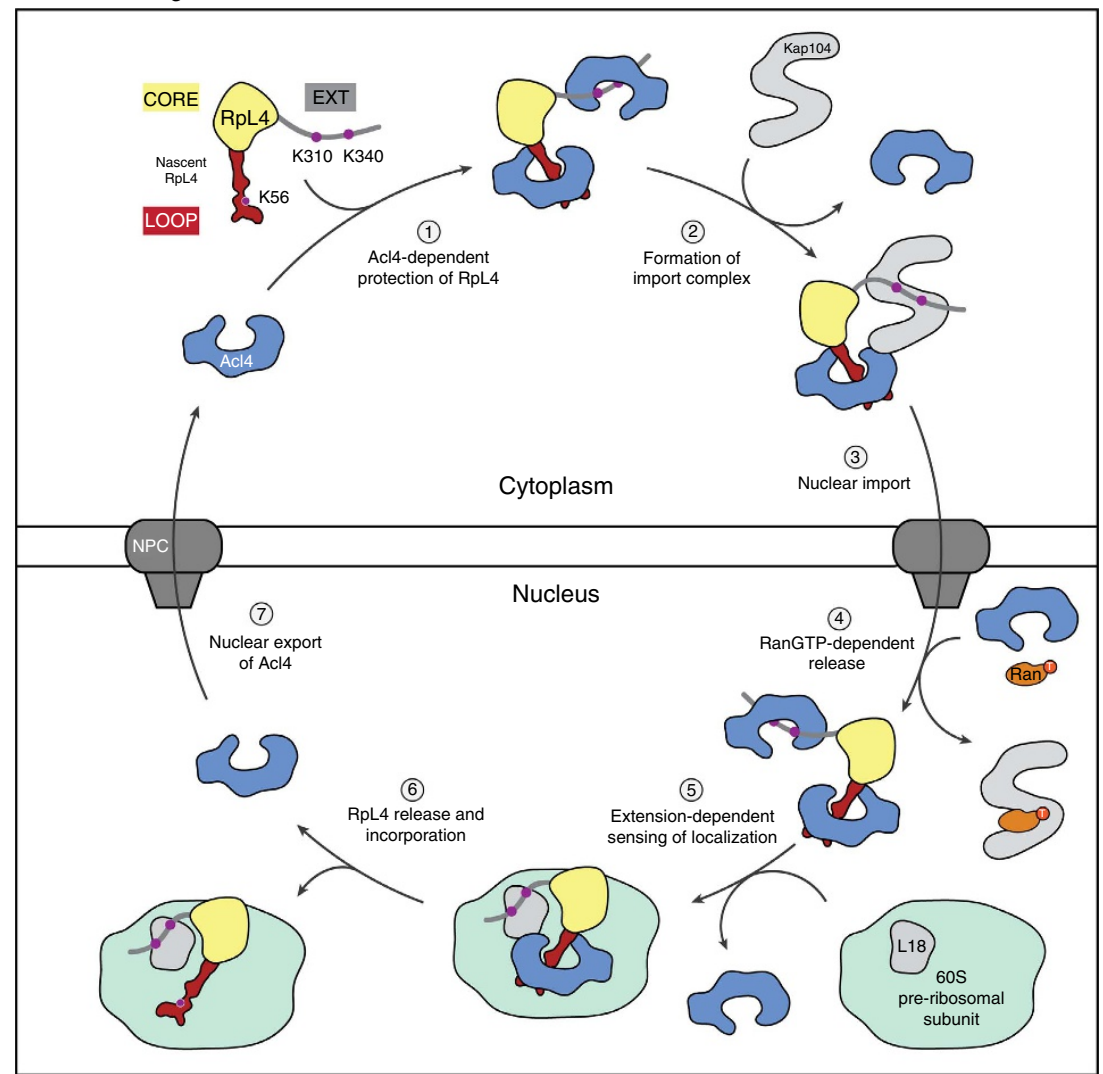

b

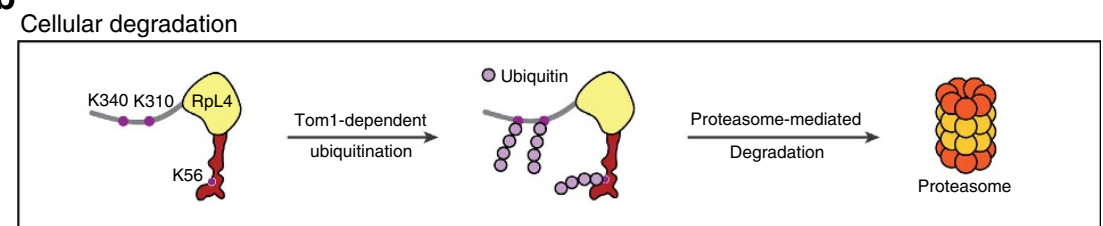

Figure 5 | Model for nuclear import and balancing of RpL4. (a) Acl4- and Kap104-mediated nuclear import of RpL4. The cycle involves seven steps. (1) Following translation, nascent RpL4 is protected by two copies of Acl4 at its unstructured loop and at the unstructured C-terminal extension.

(2) A stoichiometric hetero-trimeric nuclear import complex is formed by binding of Acl4•RpL4 to the transport factor Kap104. Kap104 binding occurs in a bi-partite fashion and involves the basic unstructured N-terminal region of Acl4 and RpL4 EXT, displacing the RpL4 ${ }^{\text {EXT }}$-bound Acl4 copy. (3) Kap104

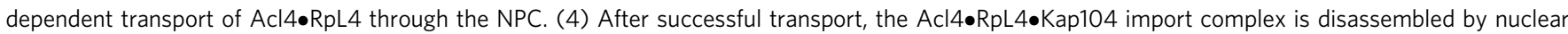
RanGTP, releasing Acl4•RpL4 into the nucleoplasm. (5) RpL4 ${ }^{\mathrm{EXT}}$ contacts RpL18 and expansion segment 7 on the surface of the pre-60S subunit ${ }^{10}$. (6) Constructive interactions result in disassembly of the Acl4•RpL4 complex and incorporation of RpL4 into the large pre-ribosomal subunit. (7) Potential nuclear export of Acl4 allows its entering into the next RpL4 transport cycle. (b) Balancing of excess unassembled ribosomal proteins. In the absence of Acl4 and Kap104, unassembled RpL4 is ubiquitinated by Tom1 and degraded by the proteasome-dependent degradation machinery ${ }^{19}$.

and resuspended in a buffer containing $20 \mathrm{mM}$ TRIS (pH 8.0), $500 \mathrm{mM} \mathrm{NaCl}$, $5 \mathrm{mM} \beta$-mercaptoethanol ( $\beta$-ME), $2 \mu \mathrm{M}$ bovine lung aprotinin (Sigma) and complete EDTA-free protease inhibitor cocktail (Roche) and flash frozen in liquid nitrogen. Cells were supplemented with $1 \mathrm{mg}$ DNase I (Roche), lysed with a cell disrupter (Avestin) and centrifuged at $4^{\circ} \mathrm{C}$ with $40,000 \mathrm{~g}$ for $1 \mathrm{~h}$. The supernatant was filtered through a $0.45 \mu \mathrm{m}$ filter (Millipore).

Purification of $\mathrm{His}_{6}$-SUMO-tagged Acl4, RpL4, Acl4•RpL4 and Kap- $\alpha$ variants. Filtered lysate of $\mathrm{His}_{6}-\mathrm{SUMO}$ tagged proteins was applied to a Ni-NTA column (Qiagen) equilibrated with a buffer containing $20 \mathrm{mM}$ TRIS (pH 8.0), $500 \mathrm{mM}$ $\mathrm{NaCl}$ and $5 \mathrm{mM} \beta-\mathrm{ME}$ and eluted with a linear imidazole gradient. Proteincontaining fractions were pooled and cleaved with ubiquitin-like-specific protease 1 (ULP1) and dialysed against a buffer containing $20 \mathrm{mM}$ TRIS (pH 8.0), $100 \mathrm{mM}$ $\mathrm{NaCl}$ and $5 \mathrm{mM} \beta$-ME (-His 6 -SUMO-RpL4 ${ }^{\mathrm{EXT}}$ was dialysed but not treated with ULP1). Dialysed proteins were applied to a Ni-NTA column equilibrated with a buffer containing $20 \mathrm{mM}$ TRIS (pH 8.0), $100 \mathrm{mM} \mathrm{NaCl}$ and $5 \mathrm{mM} \beta$-ME and the unbound fraction was loaded onto a HiTrapQ HP (GE Healthcare) ion exchange column equilibrated in a buffer containing $20 \mathrm{mM}$ TRIS (pH 8.0), $100 \mathrm{mM} \mathrm{NaCl}$, and $5 \mathrm{mM}$ DTT and eluted with a linear salt gradient. Protein-containing fractions were pooled, concentrated and injected on a 16/60 HiLoad Superdex 200 size exclusion column (GE Healthcare) equilibrated with a buffer containing $20 \mathrm{mM}$ TRIS ( $\mathrm{pH} 8.0), 100 \mathrm{mM} \mathrm{NaCl}$ and $5 \mathrm{mM}$ DTT. Protein-containing fractions were pooled, concentrated to $\sim 20 \mathrm{mg} \mathrm{ml}^{-1}$ and flash frozen in liquid nitrogen for further use.

Purification of GST-tagged hsKap104, Kap104 and Acl4. Cleared cell lysate of proteins with N-terminal Glutathione-S-transferase (GST) tag was applied to a glutathione sepharose column equilibrated with a buffer containing $20 \mathrm{mM}$ TRIS (pH 8.0), $100 \mathrm{mM} \mathrm{NaCl}$ and $5 \mathrm{mM}$ DTT and eluted with a linear gradient of reduced glutathione. Pooled fractions were cleaved with PreScission protease (GE Healthcare) for at least $10 \mathrm{~h}$ and dialysed against a buffer containing $20 \mathrm{mM}$ TRIS (pH 8.0), $100 \mathrm{mM} \mathrm{NaCl}$ and $5 \mathrm{mM}$ DTT (GST-Kap104 and GST-Acl4 for subsequent GST pull-downs were dialysed but not treated with PreScission protease). Dialysed proteins were bound to a HiTrapQ HP (GE Healthcare) ion exchange column equilibrated in a buffer containing $20 \mathrm{mM}$ TRIS ( $\mathrm{pH} 8.0$ ), $100 \mathrm{mM} \mathrm{NaCl}$ and $5 \mathrm{mM}$ DTT and eluted with a linear salt gradient. Protein-containing fractions were pooled, concentrated and injected on a 16/60 HiLoad Superdex 200 size exclusion column (GE Healthcare) equilibrated with a buffer containing $20 \mathrm{mM}$ TRIS (pH 8.0), $100 \mathrm{mM} \mathrm{NaCl}$ and $5 \mathrm{mM}$ DTT. Protein-containing fractions were pooled, concentrated to $\sim 20 \mathrm{mg} \mathrm{ml}^{-1}$ and flash frozen in liquid nitrogen for further use. 
Purification of Ran ${ }^{\mathrm{Q} 69 \mathrm{~L}}$. Cleared lysate of $\mathrm{His}_{6}-\mathrm{Ran}^{\mathrm{Q} 69 \mathrm{~L}}$ was applied to a Ni-NTA column (Qiagen) equilibrated with a buffer containing $20 \mathrm{mM}$ TRIS (pH 8.0), $500 \mathrm{mM} \mathrm{NaCl}$ and $5 \mathrm{mM} \beta$-ME and eluted with a linear imidazole gradient. Pooled fractions were cleaved with PreScission protease (GE Healthcare) for at least $10 \mathrm{~h}$ and dialysed against a buffer containing $20 \mathrm{mM}$ TRIS ( $\mathrm{pH} 8.0$ ), $100 \mathrm{mM} \mathrm{NaCl}$ and $5 \mathrm{mM}$ DTT. Cleaved protein was bound to a HiTrapQ HP (GE Healthcare) ion exchange column equilibrated in a buffer containing $20 \mathrm{mM}$ TRIS ( $\mathrm{pH} 8.0$ ), $100 \mathrm{mM} \mathrm{NaCl}$ and $5 \mathrm{mM}$ DTT and eluted with a linear salt gradient. Protein-containing fractions were pooled, concentrated and injected on a 16/60 HiLoad Superdex 75 size exclusion column (GE Healthcare) equilibrated with a buffer containing $20 \mathrm{mM}$ TRIS (pH 8.0), $100 \mathrm{mM} \mathrm{NaCl}$ and $5 \mathrm{mM}$ DTT. Purified Ran ${ }^{\text {Q69L }}$ was charged with GTP by incubation with $10 \mathrm{mM}$ EDTA and $2 \mathrm{mM} \mathrm{GTP}$ for $30 \mathrm{~min}$ at $4{ }^{\circ} \mathrm{C}$. Nucleotide exchange was stopped by the addition of $25 \mathrm{mM}$ $\mathrm{MgCl}_{2}$ (ref. 23). $\operatorname{Ran}^{\mathrm{Q} 69 \mathrm{~L}}$ GTP was injected on a Superdex 200 10/300 GL size exclusion column (GE Healthcare) pre-equilibrated with a buffer containing $20 \mathrm{mM}$ TRIS (pH 8.0), $100 \mathrm{mM} \mathrm{NaCl}, 5 \mathrm{mM}$ DTT and $5 \mu \mathrm{M}$ GTP. Proteincontaining fractions were pooled, concentrated to $\sim 20 \mathrm{mg} \mathrm{ml}^{-1}$ and flash frozen in liquid nitrogen for further use.

Purification of Acl $\bullet$ RpL4. His ${ }_{6}$-SUMO-tagged Acl4 and $\mathrm{His}_{6}$-SUMO-tagged RpL4, encompassing residues 28-361 and 1-277, respectively, were coexpressed, as previously described ${ }^{10}$. Filtered lysate was applied to a Ni-NTA column (Qiagen) equilibrated with a buffer containing $20 \mathrm{mM}$ TRIS (pH 8.0), $500 \mathrm{mM} \mathrm{NaCl}$ and $5 \mathrm{mM} \beta$-ME and eluted with a linear imidazole gradient. Protein-containing fractions were pooled and cleaved with ULP1 and dialysed against a buffer containing $20 \mathrm{mM}$ TRIS (pH 8.0), $100 \mathrm{mM} \mathrm{NaCl}$ and $5 \mathrm{mM} \beta$-ME. Dialysed proteins were applied to a Ni-NTA column equilibrated with a buffer containing $20 \mathrm{mM}$ TRIS (pH 8.0), $100 \mathrm{mM} \mathrm{NaCl}$ and $5 \mathrm{mM} \beta$-ME and the unbound fraction was loaded onto a HiTrapQ HP (GE Healthcare) ion exchange column equilibrated in a buffer containing $20 \mathrm{mM}$ TRIS (pH 8.0), $100 \mathrm{mM} \mathrm{NaCl}$ and $5 \mathrm{mM}$ DTT and eluted with a linear salt gradient. Protein-containing fractions were pooled, concentrated and injected on a 16/60 HiLoad Superdex 200 size exclusion column (GE Healthcare) equilibrated with a buffer containing $20 \mathrm{mM}$ TRIS (pH 8.0), $100 \mathrm{mM} \mathrm{NaCl}$ and $5 \mathrm{mM}$ DTT. Protein-containing fractions were pooled, concentrated to $\sim 20 \mathrm{mg} \mathrm{ml}^{-1}$ and used for crystallization.

Purification of hsKap104•RpL4 ${ }^{\mathrm{EXT}}$. hsKap104 and RpL4 $4^{\mathrm{EXT}}$, encompassing residues $308-332$, were purified individually, as described above. The $h$ SKap104•RpL4 ${ }^{\text {EXT }}$ complex was assembled on a 16/60 HiLoad Superdex 200 size exclusion column (GE Healthcare) equilibrated with a buffer containing $20 \mathrm{mM}$ TRIS (pH 8.0), $100 \mathrm{mM} \mathrm{NaCl}$ and $5 \mathrm{mM}$ DTT. Complex assembly was carried out in the presence of a 5-fold molar excess of $\mathrm{RpL} 4^{\mathrm{EXT}}$ over $h s$ Kap104 to yield a stoichiometric $h s K a p 104 \bullet R p L 4{ }^{\mathrm{EXT}}$ complex. Protein-containing fractions were pooled, concentrated to $\sim 20 \mathrm{mg} \mathrm{ml}^{-1}$ and used for crystallization.

Structure determination and refinement of Acl4•RpL4. Crystals of the $C$. thermophilum Acl4•RpL4 complex, encompassing residues 28-361 and 1-277, respectively, were obtained by hanging drop vapour diffusion at $21^{\circ} \mathrm{C}$ using $1 \mu \mathrm{l}$ protein solution and $1 \mu \mathrm{l}$ reservoir solution containing 0.1 M BIS-TRIS ( $\mathrm{pH}$ 5.5), $2 \%(\mathrm{v} / \mathrm{v}$ ) Tacsimate ( $\mathrm{pH} 5.5)$, and $13 \%$ (w/v) PEG 3350. Acl4•RpL4 crystals grew in the orthorhombic space group $\mathrm{P} 2{ }_{1} 2_{1} 2$ at a protein concentration of $17.5 \mathrm{mg} \mathrm{ml}^{-1}$ and reached their maximum size of $\sim 100 \times 50 \times 50 \mu \mathrm{m}^{3}$ within one week. Cryo protection of the crystals was achieved with $0.1 \mathrm{M}$ BIS-TRIS (pH 5.5), 2\% (v/v) Tacsimate ( $\mathrm{pH} 5.5$ ), 15\% (w/v) PEG 3350 and 20\% (v/v) ethylene glycol added in $5 \%$ increments. Collection of X-ray diffraction data was performed at $100 \mathrm{~K}$ at beamline BL12-2 at the Stanford Synchrotron Radiation Lightsource (SSRL) and crystals diffracted to a resolution of $2.4 \AA$. X-ray data were processed using XDS (ref. 24). The structure of the Acl $4 \bullet$ RpL4 complex was solved by single-wavelength anomalous dispersion (SAD) using anomalous scattering data collected at the selenium edge of SeMet-labelled crystals. Eight selenium sites were identified with SHELXD and initial phases were calculated with SHARP ${ }^{25,26}$. Density modification with solvent flattening and histogram matching was performed using DM (ref. 27). The initial electron density map was of high-quality and allowed for building of a complete model of the Acl4•RpL4 complex. A final model of the complex was generated by iterative rounds of model building and refinement in Coot and PHENIX, consisting of Acl4 residues 28-361 and RpL4 residues 4-272 (refs 28,29). No electron density was observed for RpL4 residues $1-3,78-88,189-202$ and 273-277, which are presumed to be disordered. The structure was refined to $R_{\text {work }}$ and $R_{\text {free }}$ values of $19.1 \%$ and $22.7 \%$, respectively. The Acl $4 \bullet$ RpL 4 model possesses excellent stereochemical parameters with no residues in disallowed regions of the Ramachandran plot as determined by MolProbity ${ }^{30}$. Further details of the data collection and refinement statistics are summarized in Table 1.

Structure determination and refinement of $\boldsymbol{h s K a p 1 0 4 \bullet R p L 4}{ }^{\text {EXT }}$. Crystals of the $h s K a p 104 \bullet R p L 4{ }^{\mathrm{EXT}}$ complex were obtained by hanging drop vapour diffusion at $21^{\circ} \mathrm{C}$ using $1 \mu \mathrm{l}$ protein solution and $1 \mu \mathrm{l}$ reservoir solution containing $0.1 \mathrm{M}$ BIS-TRIS-propane ( $\mathrm{pH} 7.0$ ) and $0.5 \mathrm{M}$ sodium citrate tribasic. hsKap104•RpL4 ${ }^{\mathrm{EXT}}$ crystals grew in the orthorhombic space group $\mathrm{P} 2{ }_{1} 2_{1} 2$ at a protein concentration of $5 \mathrm{mg} \mathrm{ml}^{-1}$ and reached their maximum size of $\sim 100 \times 50 \times 50 \mu \mathrm{m}^{3}$ within one week. Cryo protection of the crystals was achieved with $0.1 \mathrm{M}$ BIS-TRIS-propane $(\mathrm{pH} 7.0)$ and $0.5 \mathrm{M}$ sodium citrate tribasic and $24 \%(\mathrm{v} / \mathrm{v})$ glycerol added in $8 \%$ increments. Collection of X-ray diffraction data was performed at $100 \mathrm{~K}$ at beamline BL12-2 at the Stanford Synchrotron Radiation Lightsource (SSRL) and crystals diffracted to a resolution of 3.0 ^. X-ray data were processed using XDS (ref. 24). The structure of the $h s$ Kap 104 $\bullet \mathrm{RpL} 4^{\mathrm{EXT}}$ complex was solved by molecular replacement using the coordinates of $h s$ Kap- $\beta 2$ (PDB ID 4JLQ) as a search model in phaser ${ }^{28,31}$. The initial electron density map was of high quality and allowed for building of a complete model of the Kap104•RpL4 ${ }^{\mathrm{EXT}}$ complex. A final model of the complex was generated by iterative rounds of model building and refinement in Coot and PHENIX, consisting of $h s$ Kap104 residues 1-890 and $\mathrm{RpL}^{\mathrm{EXT}}$ residues 326-332 (refs 28,29). No electron density was observed for $h$ SKap104 residues 1-4 and 321-367 and for RpL4 ${ }^{\mathrm{EXT}}$ residues 308-325, which are presumed to be disordered. The structure was refined to $R_{\text {work }}$ and $R_{\text {free }}$ values of $20.8 \%$ and $23.8 \%$, respectively. The $h s \mathrm{Kap} 104 \bullet \mathrm{RpL} 44^{\mathrm{EXT}}$ model possesses excellent stereochemical parameters with no residues in the disallowed regions of the Ramachandran plot as determined by MolProbity ${ }^{30}$. Further details of the data collection and refinement statistics are summarized in Table 1.

GST pull-down interaction analysis. Interaction studies with GST-Acl4 coexpressed with SUMO-RpL4 ${ }^{\Delta \mathrm{EXT}}$ were performed using GST pull-down experiments. Approximately $100 \mu \mathrm{l}$ glutathione-coupled sepharose beads (GE Healthcare) were equilibrated with a buffer containing $20 \mathrm{mM}$ TRIS ( $\mathrm{pH} 8.0$ ), $100 \mathrm{mM} \mathrm{NaCl}$ and $5 \mathrm{mM}$ DTT and were incubated with cleared and filtered lysate from 11 bacterial expression culture for $1 \mathrm{~h}$ at $4{ }^{\circ} \mathrm{C}$. GST-beads were washed three times with $15 \mathrm{ml}$ buffer containing $20 \mathrm{mM}$ TRIS (pH 8.0), $100 \mathrm{mM} \mathrm{NaCl}$ and $5 \mathrm{mM}$ DTT and centrifuged with $500 \mathrm{~g}$ at $4{ }^{\circ} \mathrm{C}$. Bound protein was eluted from the beads with $250 \mu \mathrm{l}$ buffer containing $20 \mathrm{mM}$ TRIS (pH 8.0), $100 \mathrm{mM} \mathrm{NaCl}, 5 \mathrm{mM}$ DTT and $25 \mathrm{mM}$ reduced glutathione. Eluted protein was resolved on a $12.5 \%$ SDS-polyacrylamide gel electrophoresis (SDS-PAGE) gel followed by visualization with Coomassie Brilliant Blue staining.

For GST pull-down experiments with pre-purified proteins, $20 \mu \mathrm{l}$ of glutathione-coupled sepharose beads were equilibrated with a buffer containing $20 \mathrm{mM}$ TRIS (pH 8.0), $100 \mathrm{mM} \mathrm{NaCl}$ and $5 \mathrm{mM} \mathrm{DTT}$ and incubated for $1 \mathrm{~h}$ with $35 \mathrm{nmol}$ GST-tagged bait proteins and untagged prey proteins. After incubation, the beads were washed four times with $200 \mu \mathrm{l}$ buffer containing $20 \mathrm{mM}$ TRIS (pH 8.0), $100 \mathrm{mM} \mathrm{NaCl}$ and $5 \mathrm{mM}$ DTT and centrifuged with $500 \mathrm{~g}$ at $4{ }^{\circ} \mathrm{C}$. SDS-sample buffer was added to the beads, followed by boiling at $95^{\circ} \mathrm{C}$ for $5 \mathrm{~min}$ and centrifugation at $30,000 \mathrm{~g}$ for $5 \mathrm{~min}$. Samples were resolved on a $12.5 \%$ SDS-PAGE gel and visualized with Coomassie Brilliant Blue staining.

Size exclusion chromatography interaction analysis. Purified Acl4, RpL4, Kap104 and Kap- $\alpha$ variants were analysed by size exclusion chromatography (SEC). Samples were injected on a Superdex 200 10/300 GL size exclusion column (GE Healthcare) pre-equilibrated with a buffer containing $20 \mathrm{mM}$ TRIS ( $\mathrm{pH} 8.0$ ), $100 \mathrm{mM} \mathrm{NaCl}$ and $5 \mathrm{mM}$ DTT. Pre-incubation was performed for $1 \mathrm{~h}$ at $4{ }^{\circ} \mathrm{C}$ before injection on a size exclusion column. Protein containing fractions were resolved on a 12.5\% SDS-PAGE gel and visualized with Coomassie Brilliant Blue staining.

Yeast analysis. All yeast media was prepared and Lithium-acetate driven S. cerevisiae transformations were performed according to standard protocols. The $S$. cerevisiae acl44 strain was generated by replacing the Acl4 gene with a kanMX4 cassette by homologous recombination, as previously described ${ }^{32}$. Details of yeast expression vectors are summarized in Supplementary Table 2.

Growth analysis and fluorescence microscopy. The growth analysis was performed in S. cerevisiae acl44 strains that were transformed with pRS415 constructs carrying various mCherry-tagged Acl4 variants. Transformed cells were selected twice on SDC-LEU plates, before analysis. Ten-fold dilution series were prepared and $17.5 \mu \mathrm{l}$ were spotted on SDC-LEU plates and grown at 23,30 and $37^{\circ} \mathrm{C}$ for $2-3$ days. Localization assays were performed using pRS415 vectors carrying mCherry-tagged Acl4 variants and a pRS413 vector harbouring eGFP-tagged RpL4. Transformed cells were selected twice on SDC-LEU-HIS plates before analysis. The variants were grown in SDC-LEU-HIS medium at $30^{\circ} \mathrm{C}$ to mid-log phase. For heat-shock analysis, cells were grown at $30^{\circ} \mathrm{C}$ to mid-log phase before shifting cells to $37^{\circ} \mathrm{C}$ for $6 \mathrm{~h}$. For fluorescence microscopy $1 \mathrm{ml}$ of cells was centrifuged at $500 \mathrm{~g}$ and washed once with $1 \mathrm{ml}$ of water. The cell pellet was resuspended in $100 \mu \mathrm{l}$ water and $10 \mu \mathrm{l}$ were analysed using a Carl Zeiss Observer Z.1 equipped with a Hamamatsu C10600 Orca-R2 camera.

Western blot analysis. In vivo Acl4 expression levels were tested by transformation of S. cerevisiae acl44 strains with pRS415 constructs carrying various HA-mCherry-tagged Acl4 variants. Transformed cells were selected twice on SDC-LEU plates, before analysis. Protein extraction from cells was performed via $\mathrm{NaOH}$ and TCA treatment ${ }^{33}$. Specifically, cells were grown at $30^{\circ} \mathrm{C}$ to an OD of $\sim 1.0$ before harvesting of $1 \mathrm{ml}$ of culture. Cell pellets were resuspended and vortexed in $1 \mathrm{ml}$ of a solution containing $1.85 \mathrm{M} \mathrm{NaOH}$ and $7.4 \%(\mathrm{v} / \mathrm{v}) \beta-\mathrm{ME}$ before incubation for $10 \mathrm{~min}$ on ice. Proteins were precipitated by addition of $150 \mu \mathrm{l}$ of $50 \%$ (w/v) TCA and incubation for $10 \mathrm{~min}$ on ice, followed by centrifugation at $30,000 \mathrm{~g}$ for $2 \mathrm{~min}$. The pellet was washed twice with $1 \mathrm{ml}$ of icecold acetone and air-dried at room temperature before resuspension in SDS 
loading buffer. Western blot analysis was performed with a rabbit anti-hexokinase antibody (US Biological; H2035-02; 1:10,000 dilution), an anti-rabbit antibody fused to an IR800 fluorescent probe (Licor, 926-32211; 1:5,000 dilution), a mouse anti-HA antibody (Covance; MMS-101P; 1:5,000 dilution), and an anti-mouse antibody coupled to alkaline phosphatase (Promega; S3721; 1:5,000 dilution). Antibodies were diluted in TBS-T supplemented with $5 \%(\mathrm{w} / \mathrm{v})$ milk powder and washes were carried out in TBS-T.

Animation of Acl4•RpL4 disassembly. Acl4 apo (PDB ID 4YNV) and ribosomebound RpL4 (PDB ID 4V88) were superposed with Acl4•RpL4 structure as reference ${ }^{10,17}$. Morphing of the Acl4•RpL4 complex into the open Acl 4 apo state and ribosome-bound RpL4 was animated using PyMOL (www.pymolorg).

Illustrations and figures. Size exclusion chromatography profiles were generated with IGOR (WaveMetrics) and assembled with Adobe Illustrator. All structure figures were generated with PyMOL (www.pymol.org). Sequence alignments were generated using ClustalX and coloured with ALSCRIPT ${ }^{34,35}$. Electrostatic potentials were calculated with APBS (Adaptive Poisson-Boltzmann Solver) software ${ }^{36}$.

Data availability. The coordinates and structure factors have been deposited with the Protein Data Bank with accession codes 5TQB (Acl4•RpL4) and 5TQC $\left(\right.$ Kap104•RpL4 $\left.{ }^{\mathrm{EXT}}\right)$. The data that support the findings of this study are available from the corresponding author on request.

\section{References}

1. Kressler, D., Hurt, E. \& Bassler, J. Driving ribosome assembly. Biochim. Biophys. Acta 1803, 673-683 (2010).

2. Johnson, A. W. Ribosomes: lifting the nuclear export ban. Curr. Biol. 24, R127-R129 (2014).

3. Lafontaine, D. L. A 'garbage can' for ribosomes: how eukaryotes degrade their ribosomes. Trends Biochem. Sci. 35, 267-277 (2010).

4. Lafontaine, D. L. Noncoding RNAs in eukaryotic ribosome biogenesis and function. Nat. Struct. Mol. Biol. 22, 11-19 (2015).

5. Roberts, P. et al. Piecemeal microautophagy of nucleus in Saccharomyces cerevisiae. Mol. Biol. Cell 14, 129-141 (2003).

6. Gorenstein, C. \& Warner, J. R. Synthesis and turnover of ribosomal proteins in the absence of 60S subunit assembly in Saccharomyces cerevisiae. Mol. Gen. Genet. 157, 327-332 (1977).

7. Warner, J. R. In the absence of ribosomal RNA synthesis, the ribosomal proteins of HeLa cells are synthesized normally and degraded rapidly. J. Mol. Biol. 115, 315-333 (1977).

8. Fromont-Racine, M., Senger, B., Saveanu, C. \& Fasiolo, F. Ribosome assembly in eukaryotes. Gene 313, 17-42 (2003).

9. Kressler, D. et al. Synchronizing nuclear import of ribosomal proteins with ribosome assembly. Science 338, 666-671 (2012).

10. Stelter, P. et al. Coordinated Ribosomal L4 Protein Assembly into the Pre-Ribosome Is Regulated by Its Eukaryote-Specific Extension. Mol. Cell 58, 854-862 (2015).

11. Pausch, P. et al. Co-translational capturing of nascent ribosomal proteins by their dedicated chaperones. Nat. Commun. 6, 7494 (2015).

12. Koch, B. et al. Yarl protects the ribosomal protein Rps3 from aggregation. J. Biol. Chem. 287, 21806-21815 (2012).

13. Eisinger, D. P., Dick, F. A., Denke, E. \& Trumpower, B. L. SQT1, which encodes an essential WD domain protein of Saccharomyces cerevisiae, suppresses dominant-negative mutations of the ribosomal protein gene QSR1. Mol. Cell Biol. 17, 5146-5155 (1997).

14. Holzer, S., Ban, N. \& Klinge, S. Crystal structure of the yeast ribosomal protein rpS3 in complex with its chaperone Yar1. J. Mol. Biol. 425, 4154-4160 (2013).

15. Calvino, F. R. et al. Symportin 1 chaperones $5 \mathrm{~S}$ RNP assembly during ribosome biogenesis by occupying an essential rRNA-binding site. Nat. Commun. 6, 6510 (2015).

16. Pillet, B. et al. The Dedicated Chaperone Acl4 Escorts Ribosomal Protein Rpl4 to Its Nuclear Pre-60S Assembly Site. PLoS Genet. 11, e1005565 (2015).

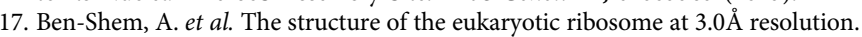
Science 334, 1524-1529 (2011).

18. Zhang, Y., Wolfle, T. \& Rospert, S. Interaction of nascent chains with the ribosomal tunnel proteins Rpl4, Rpl17, and Rpl39 of Saccharomyces cerevisiae. J. Biol. Chem. 288, 33697-33707 (2013).

19. Sung, M. K. et al. A conserved quality-control pathway that mediates degradation of unassembled ribosomal proteins. Elife 5, e19105 (2016).

20. Lee, B. J. et al. Rules for nuclear localization sequence recognition by karyopherin beta 2. Cell 126, 543-558 (2006).

21. Mossessova, E. \& Lima, C. D. Ulp1-SUMO crystal structure and genetic analysis reveal conserved interactions and a regulatory element essential for cell growth in yeast. Mol. Cell 5, 865-876 (2000).

22. Hoelz, A., Nairn, A. C. \& Kuriyan, J. Crystal structure of a tetradecameric assembly of the association domain of $\mathrm{Ca} 2+/$ calmodulin-dependent kinase II. Mol. Cell 11, 1241-1251 (2003).
23. Maertens, G. N. et al. Structural basis for nuclear import of splicing factors by human Transportin 3. Proc. Natl. Acad. Sci. USA 111, 2728-2733 (2014).

24. Kabsch, W. Xds. Acta Crystallogr. D 66, 125-132 (2010).

25. Bricogne, G., Vonrhein, C., Flensburg, C., Schiltz, M. \& Paciorek, W. Generation, representation and flow of phase information in structure determination: recent developments in and around SHARP 2.0. Acta Crystallogr. D 59, 2023-2030 (2003).

26. Sheldrick, G. M. A short history of SHELX. Acta Crystallogr. A 64, 112-122 (2008).

27. Bailey, S. The Ccp4 Suite-Programs for Protein Crystallography. Acta Crystallogr. D 50, 760-763 (1994).

28. Adams, P. D. et al. PHENIX: a comprehensive Python-based system for macromolecular structure solution. Acta Crystallogr. D 66, 213-221 (2010).

29. Emsley, P. \& Cowtan, K. Coot: model-building tools for molecular graphics. Acta Crystallogr. D 60, 2126-2132 (2004).

30. Davis, I. W. et al. MolProbity: all-atom contacts and structure validation for proteins and nucleic acids. Nucleic Acids Res. 35, W375-W383 (2007).

31. Soniat, M. et al. Crystal structure of human Karyopherin beta2 bound to the PY-NLS of Saccharomyces cerevisiae Nab2. J. Struct. Funct. Genomics 14, 31-35 (2013)

32. Janke, C. et al. A versatile toolbox for PCR-based tagging of yeast genes: new fluorescent proteins, more markers and promoter substitution cassettes. Yeast 21, 947-962 (2004).

33. Yaffe, M. P. \& Schatz, G. Two nuclear mutations that block mitochondrial protein import in yeast. Proc Natl Acad Sci USA 81, 4819-4823 (1984).

34. Jeanmougin, F., Thompson, J. D., Gouy, M., Higgins, D. G. \& Gibson, T. J. Multiple sequence alignment with Clustal X. Trends Biochem. Sci. 23, 403-405 (1998).

35. Barton, G. J. ALSCRIPT: a tool to format multiple sequence alignments. Protein Eng. 6, 37-40 (1993).

36. Baker, N. A., Sept, D., Joseph, S., Holst, M. J. \& McCammon, J. A. Electrostatics of nanosystems: application to microtubules and the ribosome. Proc. Natl. Acad. Sci. USA 98, 10037-10041 (2001).

\section{Acknowledgements}

We thank Daniel H. Lin, Alina Patke and Emily J. Rundlet, for critical reading of the manuscript, Raymond J. Deshaies, Min-Kyung Sung, and Andrew M. Davenport for helpful discussions, Christopher Markosian for technical support, Jens Kaiser and the scientific staff of SSRL Beamline 12-2 for their support with X-ray diffraction measurements and Yuh Min Chook and Raymond J. Deshaies for sharing material. The operations at SSRL are supported by the Department of Energy and the National Institutes of Health. We acknowledge the Gordon and Betty Moore Foundation, the Beckman Institute, and the Sanofi-Aventis Bioengineering Research Program for their support of the Molecular Observatory at the California Institute of Technology. F.M.H. was supported by a PhD fellowship of the Boehringer Ingelheim Fonds. A.H. is a Faculty Scholar of the Howard Hughes Medical Institute, an inaugural Principal Investigator of the Heritage Medical Research Institute for the Advancement of Medicine and Science at Caltech and was supported by Caltech startup funds, a Kimmel Scholar Award of the Sidney Kimmel Foundation for Cancer Research, and a Teacher-Scholar Award of the Camille \& Henry Dreyfus Foundation.

\section{Author contributions}

F.M.H. and A.H. conceived of the project, designed the experiments, analysed the data and wrote the manuscript. F.M.H. performed the experiments.

\section{Additional information}

Supplementary Information accompanies this paper at http://www.nature.com/ naturecommunications

Competing financial interests: The authors declare no competing financial interests

Reprints and permission information is available online at http://npg.nature.com/ reprintsandpermissions/

How to cite this article: Huber, F. M. \& Hoelz, A. Molecular basis for protection of ribosomal protein L4 from cellular degradation. Nat. Commun. 8, 14354 doi: $10.1038 /$ ncomms14354 (2017).

Publisher's note: Springer Nature remains neutral with regard to jurisdictional claims in published maps and institutional affiliations.

This work is licensed under a Creative Commons Attribution 4.0 International License. The images or other third party material in this article are included in the article's Creative Commons license, unless indicated otherwise in the credit line; if the material is not included under the Creative Commons license, users will need to obtain permission from the license holder to reproduce the material. To view a copy of this license, visit http://creativecommons.org/licenses/by/4.0/

(C) The Author(s) 2017 